\title{
Kontrastmittelsonografie des Abdomens
}

\author{
Christoph F. Dietrich, Christian Greis
}

\begin{abstract}
Ultraschall-Kontrastmittel werden zur weiteren Abklärung unklarer Befunde, wie z. B. Raumforderungen der Leber oder anderer Bauchorgane eingesetzt. Mit Hilfe von mikroskopisch kleinen Gasblasen als Echoverstärker kann die Durchblutung der Organe präzise dargestellt werden. Dadurch lassen sich spezielle Fragestellungen beantworten und Organveränderungen in ihrem Verlauf dokumentieren.
\end{abstract}

Darstellung von Mikrogefäßen I Mit der Entwicklung von Ultraschallkontrastmitteln (USKM, Ultrasound Contrast Agents, UCA) aus Mikrobläschen konnten wesentliche Einschränkungen des konventionellen B-Bild-Modus und auch der Doppler-Technik überwunden werden. Mit USKM lassen sich nicht nur Makro-, sondern auch Mikrogefäße darstellen. Die Anreicherungsmuster von Läsionen können somit in allen Phasen (arteriell, portalvenös, später und eventuell postvaskulär) in ähnlicher Weise untersucht werden wie in der Kontrastmittel-CT und MRT.

\section{Kontrastmittel}

Welches Kontrastmittel? I In Deutschland ist heutzutage lediglich ein Ultraschall-Kontrastmittel für die Untersuchung der Leber zugelassen. SonoVue ${ }^{\circledR}$ gilt auch im übrigen Europa heutzutage aufgrund der Zulassungscharakteristika und publizierten Evidenz als USKM (oder Echosignalverstärker) der ersten Wahl $[1,2]$ ( Abb. 1). Neben SonoVue sind auch noch die Kontrastmittel Optison und Luminity zugelassen, allerdings nur für die Kontrastierung der Herzkammern in der Echokardiografie. In Norwegen ist noch Sonazoid ${ }^{\circledR}$ zugelassen. Andere Produkte werden inzwischen nicht mehr angeboten (Levovist ${ }^{\circledR}$ ) oder haben sich nicht durchgesetzt [3].

Vorbereitung des Kontrastmittels | Zuerst wird die Spritze mit dem Lösungsmittel (0,9\% NaCl-Lösung) auf den Entnahmedorn aufgeschraubt, um das Ausweichen des Gases zu vermeiden. Danach wird dieser mit dem Glasbehälter des lyophilisierten Ultraschallkontrastmittels verbunden ( Abb. 2). Die fertigen Mikrobläschen entstehen nach Injektion des Lösungsmittels durch Schütteln über 20 Sekunden.

Die zubereitete Suspension ist milchig weiß und muss aus Sterilitätsgründen innerhalb von 6 Stunden verbraucht werden.
Bei längerem Verweilen der Suspension im Glasbehälter schwimmen die Bläschen oben auf und die darunter liegende Lösung wird klar. In diesem Falle muss die KM-Suspension vor Entnahme durch leichtes Schütteln gemischt werden.

Injektion I Für die Injektion koppeln wir die KM-Spritze direkt an die Patientenkanüle an. Ein Drei-Wege-Hahn wird von uns nicht verwendet, da die unterschiedlichen Durchmesser zu unterschiedlichen Strömungen und evtl. zur Kontrastmittelzerstörung führen können. Zuführende Schläuche sind auf ein Minimum zu beschränken. Wir benutzen eine Kanüle mit zwei Zugangswegen. Das Kochsalz darf durch den Filter gegeben werden - aber niemals das Ultraschallkontrastmittel. Auch wenn es von erfahrenen Intensivmedizinern bespottet wird, ist die Kanüle bevorzugt in der Ellenbeuge zu legen, um bestmöglich standardisierte Kreislaufzeiten zu erhalten ( $\triangleright$ Abb. $\mathbf{3})$.

Injektion von Kochsalz | Die empfohlene Bolusinjektion sollte rasch erfolgen, gefolgt von der raschen Injektion von $10 \mathrm{ml}$ Kochsalz-Lösung. Größere Kochsalzmengen sind möglich, aber er-

Abb. 1 Ultraschallkontrastmittelbox SonoVue ${ }^{\circledR}$.

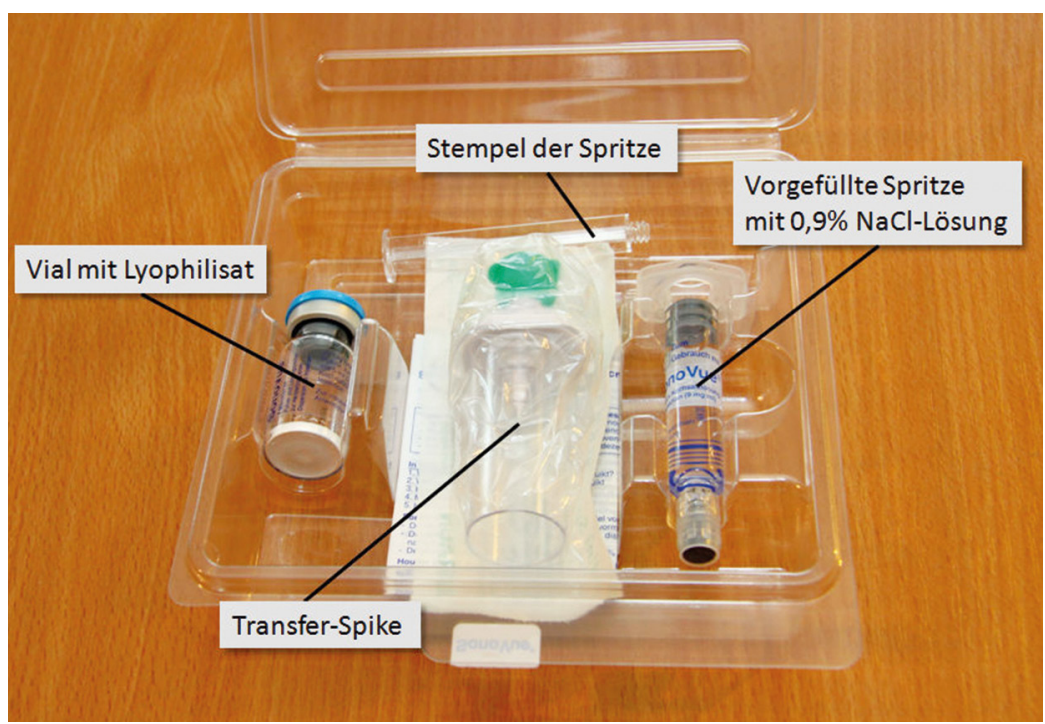


Abb. 2 Vorbereitung der Applikation. Zunächst wird die Spritze auf den Entnahmedorn aufgeschraubt und dann das Vial mit dem Lyophilisat von unten in den Dorn eingeführt, bis es hörbar einrastet. Danach kann die Kochsalzlösung in das Vial eingespritzt werden.

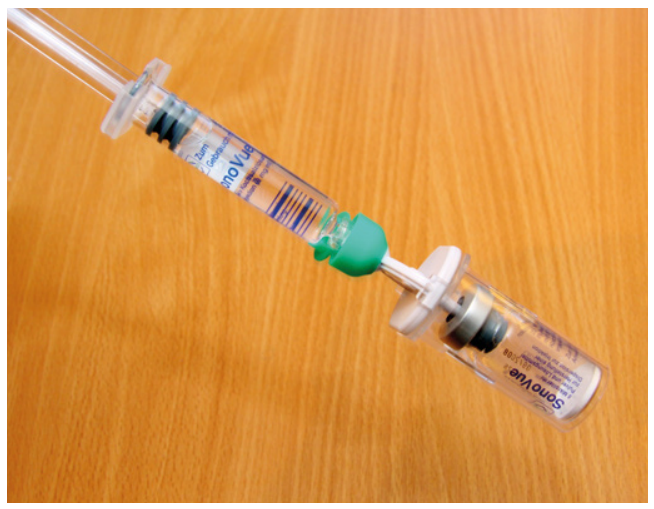

schweren den Injektionsvorgang. Einen zu hohen Injektionsdruck sollte man vermeiden, um die Bläschen nicht zu zerstören.

Dosierung | Die Dosierung des Ultraschallkontrastmittels erfolgt unter Berücksichtigung

- des Ultraschallgeräts,

- der enthaltenen Software,

- des verwendeten Schallkopfs,

- des zu untersuchenden Organs und

- der Fragestellung.

Die Möglichkeit einer erneuten Injektion sollte zur Klärung offen gebliebener Fragestellungen genutzt werden. Es hat sich bei der transkutanen Anwendung ein Bolus von 2,4 ml SonoVue ${ }^{\circledR}$ bewährt und beim endoskopischen Ultraschall $4,8 \mathrm{ml}$ (die gesamte Einheit).

Eine höhere oder zu hohe Kontrastmitteldosierung führt zu besseren Kontrasteffekten in der Spätphase und ermöglicht die teilweise Kompensation der Bläschenzerstörung durch eine zu lange Untersuchungsdauer.

Nachteil einer höheren Kontrastmitteldosis sind die Abschattung in der Tiefe, die Überstrahlung kleiner Strukturen sowie das Erreichen einer Signalsättigung, besonders bei beabsichtigter Quantifizierung ( $\triangleright$ Abb. 4 ).

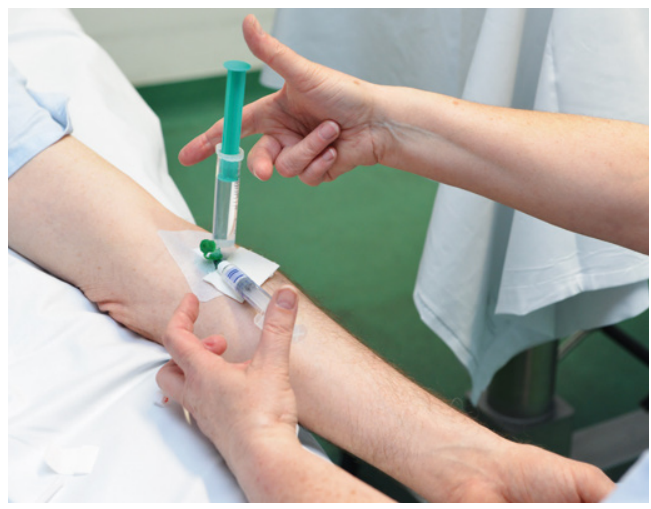

Abb. 3 Applikation des Ultraschallkontrastmittels auf der kontralateralen Seite, bevorzugt in der Ellenbeuge.

"Circle of Disaster" | Der mechanische Index (Sendeleistung) beschreibt den angenommenen Schalldruck im Fokus des Ultraschallstrahls in einem durchschnittlichen Gewebe. Er ist definiert als maximaler negativer Druck (d. h. in der Unterdruckphase) geteilt durch die Quadratwurzel der Schallfrequenz. Er ist ein Maß für bei der Schallexposition entstehenden mechanischen Kräfte im Gewebe. Eine erhöhte Sendeleistung führt zu einer verstärkten Zerstörung von KontrastmittelMikrobläschen. Dies kann durch eine Erhöhung der KM-Dosis teilweise kompensiert werden, was aber eine erhöhte Abschattung in der Tiefe (verringerte Penetration) zur Folge hat. Dadurch wird wiederum eine Erhöhung der Sendeleistung erforderlich. Der „Circle of Disaster“ schließt sich $(\checkmark$ Abb. 5). Eine gute Kontrastmittel-Untersuchung erfordert deshalb eine ausgewogene Balance dieser Parameter.

Gain I Der Gain (Verstärkung) beschreibt die Nachverstärkung des Videosignals auf dem Bildschirm. Ein hoher Gain ist kein Ersatz für ein zu schwaches Kontrastmittelsignal, da er auch Rausch- und Artefaktsignale gleichermaßen verstärkt. Der Gain sollte so eingestellt werden, dass schwache Signale annähernd schwarz und starke Signale mit fast maximaler Helligkeit dargestellt werden. So wird der gesamte dynamische Bereich des Bildschirms für die Darstellung ausgenutzt $(\triangleright$ Abb. 6).

Abb. 4 Abschattung bei zu hoher Kontrastmitteldosis. Bei zu geringer Dosis ist die Kontrastierung schwach (a), bei richtiger Dosis stark mit deutlich sichtbaren Unterschieden zwischen stark und weniger stark durchbluteten Bereichen (b), bei zu hoher Dosis überstrahlt mit distaler Abschattung (c).
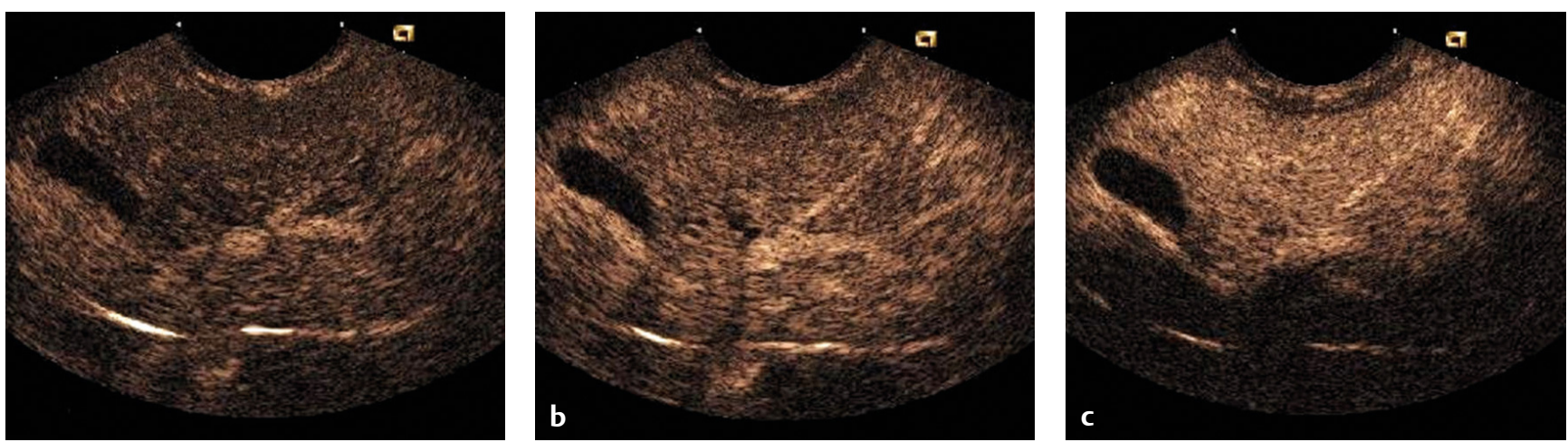
Untersucher I Wer Kontrastmittelsonografie der Leber durchführt, muss Indikationen, Kontraindikationen und Artefakte [4-7] kennen, die Notfalltherapie und die Videoclip-Dokumentation beherrschen und sollte nach EFSUMB-Empfehlung diese Methode unter Anleitung eines Level3-CEUS-Trainers erlernt haben.

\section{Untersuchung}

Beurteilung | Kontrastmittelultraschall erlaubt die dynamische Beurteilung der Kontrastmittel An- und Abflutung in allen vaskulären Phasen. Beurteilt werden die Gefäßversorgung (Vaskularisierung) und die Durchblutung (Perfusion). Der Vaskularisierungsgrad wird durch die Gefäßdichte und das relative Blutvolumen beschrieben. Bei der Leber erfolgt der Kontrastmitteleinstrom zunächst über das arterielle Gefäßsystem und dann mit einiger Verzögerung über das portalvenöse Gefäßsystem. Es müssen alle Phasen berücksichtigt werden, die zusammen das vollständige Gefäßbett repräsentieren. Die Gefäßarchitektur beschreibt die Geometrie des Gefäßverlaufs. Die Dynamik des Blutflusses beschreibt die Blutflussgeschwindigkeit und Transitzeit unter Berücksichtigung der arteriellen, portalvenösen und venösen Gefäße.

Schallpausen | Eine kontinuierliche Aufzeichnung charakteristischer (repräsentativer) Phasen mit dazwischen liegenden Schallpausen (Bild „freezen“) wird zur Dokumentation empfohlen. Eine kontinuierliche Beschallung über den Kontrastierungszeitraum führt selbst bei niedrigem mechanischen Index zu einer hohen schallinduzierten Bläschenzerstörung und ist zu vermeiden.

Voraussetzung I Wird ein Kontrastmittel-Ultraschall durchgeführt, sollten alle früheren Ergebnisse der bildgebenden Diagnostik sowie die demografischen und klinischen Daten des Patienten bekannt sein - genau wie bei der konventionellen Ultraschalluntersuchung [8, 9]. Der Patient sollte über die Notwendigkeit der Kontrastmittelapplikation sowie die Möglichkeit allergoider (pseudoallergischer) Reaktionen aufgeklärt werden (s. Nebenwirkungen). Die Anamnese bezüglich möglicher Allergien sollte erfasst werden.

\section{Ablaufschritte}

- Jede Untersuchung sollte mit konventionellen B-Bild- und Doppler-Techniken beginnen.

- Nach Identifizierung der Zielläsion wird der Schallkopf an diesem Ort gehalten und das Gerät auf einen kontrastspezifischen Modus mit niedrigem mechanischen Index umgestellt.

- Es sollte ein Schallfenster gewählt werden, bei dem die Läsion ggf. mittels geeigneter Atemmanöver möglichst gut im Bild gehalten werden kann und nahe beim Schallkopf liegt. Das Bild sollte soweit aufgezoomt werden, dass die

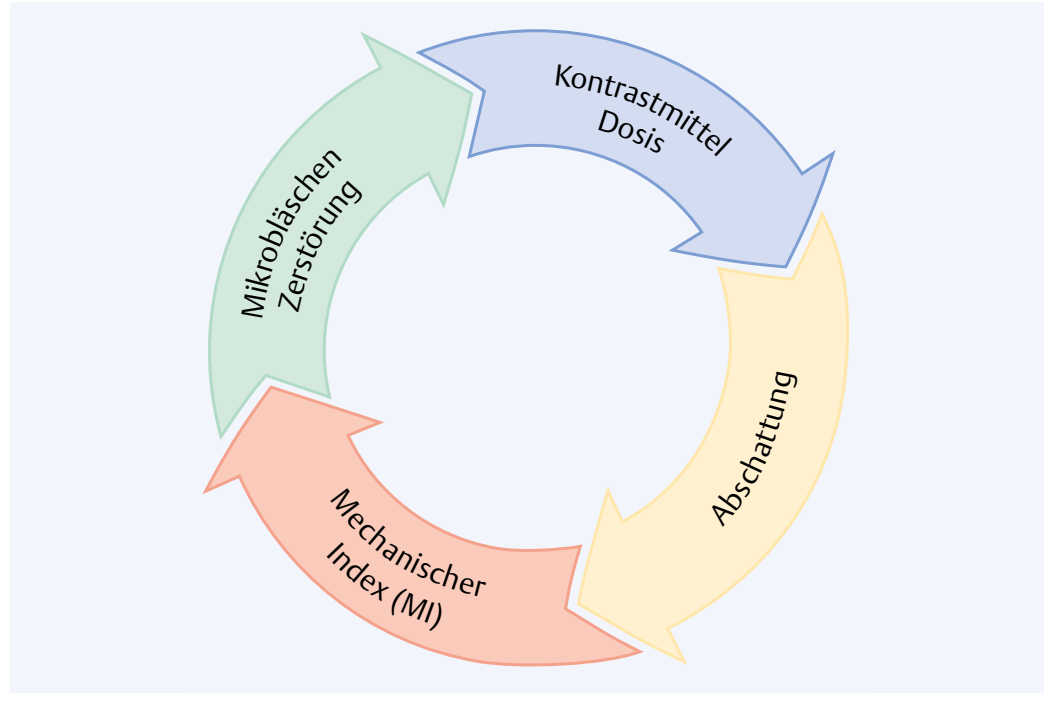

Läsion mit etwas umgebendem normalen Lebergewebe formatfüllend abgebildet ist.

- Verschiedene Techniken ermöglichen die simultane Darstellung von Gewebe- und KM-Signalen und helfen somit bei der anatomischen Orientierung (Überlagerungsverfahren, Dualbild).

- Das USKM wird als Bolus verabreicht, anschließend wird mit physiologischer Kochsalzlösung nachgespült $(10 \mathrm{ml})$.

- Idealerweise liegt der Durchmesser des venösen Zugangs nicht unter 20 G, um eine Zerstörung der Mikrobläschen während der Injektion zu minimieren. Zentralvenöse Katheter und Port-Systeme können verwendet werden, wenn kein Filter zwischengeschaltet ist, der einen hohen Injektionsdruck erfordert.

- Zum Zeitpunkt der Injektion des USKM sollte eine Stoppuhr gestartet werden.

- In jeder Phase sollten aussagekräftige Videosequenzen gespeichert werden.

- Die Injektion kann wiederholt werden, wenn die meisten Mikrobläschen verschwunden sind und das Bild wieder nahezu schwarz erscheint (nach 6-10 min).

Terminologie I In der Praxis wird das Ausmaß der Anreicherung (Enhancement) im Vergleich zum umgebenden (normalen) Lebergewebe beschrieben, wobei zusätzlich noch der Zeitpunkt (z. B. arterielle, portalvenöse oder späte Phase) angegeben werden muss: Gleichmäßige Anreicherung, minder- oder mehranreichernd. Bei der Leber muss man hierbei noch den Zeitpunkt angeben. Eine Läsion kann beispielsweise arteriell mehranreichernd, aber portalvenös minderanreichernd sein.

Bei nicht durchblutetem Gewebe (z. B. Nekrose) lässt sich keine Kontrastmittelanreicherung nachweisen. Dies ist von einem Washout einer initial kontrastierten Region zu unterscheiden. Der Begriff „echogen“ sollte für das morphologische B-Bild-Signal reserviert bleiben.
Abb. 5 Zusammenhang zwischen Sendeleistung (mechanischer Index), Mikrobläschenzerstörung, Kontrastmitteldosis und Eindringtiefe (Abschattung). 
Abb. 6 Bei geringer Gain-Einstellung werden nur sehr starke Signale dargestellt (a). Bei richtiger Gain-Einstellung werden alle Signale unter voller Ausnützung des verfügbaren Grauwertebereiches dargestellt (b). Bei zu hoher Gain-Einstellung werden bereits Signale mittlerer Intensität mit maximaler Helligkeit dargestellt und Rauschsignale werden mit erfasst. Das Bild wirkt überstrahlt und verrauscht (c).

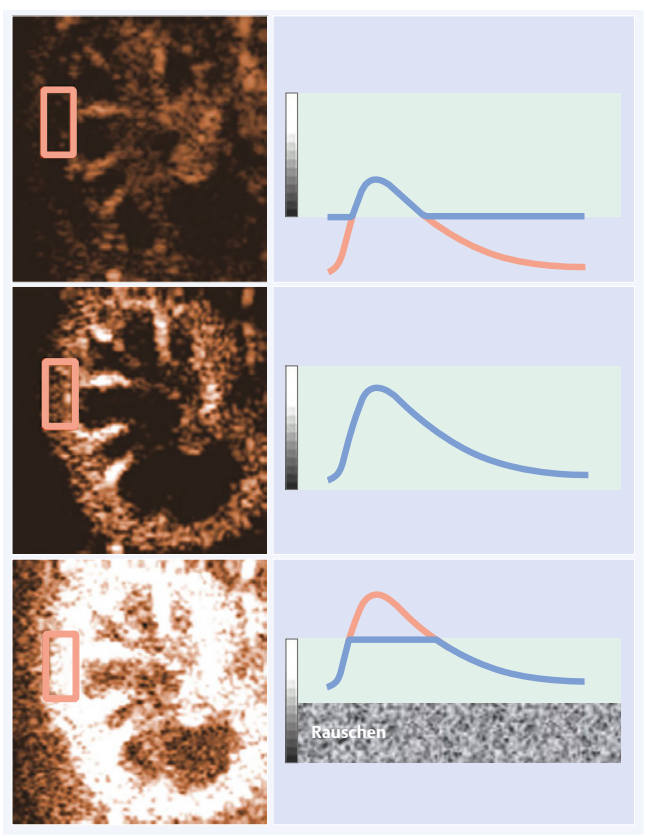

Kontraindikationen

- bekannte Überempfindlichkeit gegen das Kontrastmittel

- bekannter Rechts-Links-Shunt

- schwere pulmonale Hypertonie (pulmonalarterieller Druck $>90 \mathrm{mmHg}$ )

- unkontrollierter systemischer Hochdruck

- akutes Atemnotsyndrom

- unmittelbar im Anschluss geplante extrakorporale Stoßwellenlithotripsie (ESWL)

Limitationen beim CT sind die hohe Strahlenbelastung (CT Abdomen: $10 \mathrm{mSv}=500$ RöntgenThorax-Aufnahmen) und die Hyperthyreose (0,05\% thyreotoxische Krise). Beim MRT sind es Metall-Fremdkörper (Herzschrittmacher, Implantate, Drähte, Tätowierung, andere) und Klaustrophobie.

\section{Organuntersuchungen im Abdomen}

Unterschiede I In der Leber werden aufgrund der dualen Blutversorgung verschiedene Kontrastierungsphasen unterschieden (arterielle, portal-venöse und Spätphase). Bei anderen Organen, beispielsweise der Prostata, unterscheidet man keine arteriellen und venösen Phasen, da die Anflutung immer arteriell ist. Man spricht eher von Anflutungs- und Auswaschphase. In Abhängigkeit von der Anflutungscharakteristik der spezifischen Läsion erfolgt die Detektion entweder in der (sehr kurzen) Anflutungsphase oder in der Spätphase. Leberfremde Läsionen (z.B. Metastasen) werden in der Regel in der portalvenösen bzw Spätphase detektiert $[8,9]$. Hepatozelluläre Karzinome in der Leber entwickeln im zirrhotischen Umbau eine erhöhte arterielle Blutversorgung und werden deshalb bevorzugt in der arteriellen Phase detektiert. Läsionen in der Milz grenzen sich dagegen besonders in der späten (sinusoidalen) Phase ab.
Leber I Die duale Blutversorgung der Leber durch die A. hepatica (25-30\%) und die Pfortader (70$75 \%$ ) führt zu drei sich überlappenden vaskulären Phasen [8, 9], da die Kontrastmittelanflutung über die Pfortader im Vergleich zur Leberarterie zeitlich später erfolgt:

- Die arterielle Phase liefert Informationen über Ausmaß und Muster der arteriellen Gefäßversorgung. Abhängig vom individuellen Kreislaufzustand beginnt die arterielle Phase im Allgemeinen innerhalb von 20 s nach der KMInjektion und setzt sich bis $30-45$ s fort. Diese Phase kann sehr schnell einsetzen, und die Dokumentation der Echtzeit-Darstellung im Kontrasmittel-Ultraschall ist notwendig. Die Details der arteriellen Phase werden oft am besten in einer langsamen Wiederholung eines gespeicherten Cine-Loops sichtbar.

- Die portalvenöse Phase dauert normalerweise bis zu 2 min nach der Injektion an. Diese beiden frühen Phasen sind sich bei allen verfügbaren Kontrastmitteln sehr ähnlich $\left(\right.$ SonoVue ${ }^{\circledR}$, Definity $^{\circledR}$, Sonazoid ${ }^{\circledR}$ ).

- Die späte Phase dauert bis zur Auswaschung des Ultraschallkontrastmittels aus dem Leberparenchym und ist für etwa 4-6 min sichtbar.

Die Kontrastmittelsonografie findet in der Gastroenterologie zunehmend Anwendung und ist bei vielen Fragestellungen mit den Ergebnissen der Computertomografie und Magnetresonanztomografie vergleichbar [10].

Pankreas I Der häufigste solide Pankreastumor ist das duktale Adenokarzinom. Aufgrund der unterschiedlichen Therapien und Prognosen von Pankreastumoren ist eine initiale sichere Differenzialdiagnose anderweitiger Tumorentitäten essenziell. Dies gilt es präoperativ abzuklären, um Patienten mit alternativen Tumorentitäten vor der signifikanten Operationsletalität und -morbidität zu bewahren. Die Güte der präoperativen Diagnostik eines interdisziplinären Zentrums kann auch anhand der präoperativ richtig erkannten anderweitigen Diagnosen gewertet werden, die nicht zu einer radikalen Standardoperationstechnik führen. Dies gilt insbesondere für das asymptomatische seröse mikrozystische Pankreasadenom, das in der Regel nicht entartet und entzündliche Pankreasveränderungen wie die Autoimmunpankreatitis, die mit Kortikosteroiden behandelt wird.

Differenzialdiagnosen bei Pankreasläsionen | Differenzierte neuroendokrine Tumoren werden (altersabhängig, Grading) beobachtet oder bei Indikationsstellung (wenn möglich) pankreasgewebesparend enukleiert und sollten keiner radikalen Operationstechnik zugeführt werden [11, 12]. Wichtig ist bei der Beurteilung der Differenzialdiagnose von soliden Pankreastumoren, dem klinischen „workflow“ mehr Bedeutung zuzumessen. So stellen metastasierte Pankreaskarzinome den Kliniker selten vor differenzialdiag- 
nostische Probleme. Das (auch histologisch) hypovaskularisierte duktale Adenokarzinom kann heutzutage mit einer relativ hohen Sicherheit von anderweitigen Neoplasien mittels kontrastverstärkter sonografischer Techniken differenziert werden.

OP-Indikation | Bei sicherem Nachweis eines Pankreastumors und dringendem Verdacht auf ein duktales Adenokarzinom des Pankreas ohne Nachweis von Fernmetastasen und bestehender kurativer Operabilität ist allerdings primär die Operation indiziert und keine Punktion mit histologischer Sicherung der Diagnose notwendig. Die Computertomografie ermöglicht in vielen Fällen dieses Leitlinien-gerechte Vorgehen. Lymphome und Metastasen (mit Ausnahme von Solitärmetastasen eines Nierenzellkarzinoms) werden im Regelfall nicht operativ behandelt [12]. Auch zystische Pankreastumoren werden heutzutage häufiger beobachtet als bisher angenommen.

Die Abklärung zystischer Pankreastumoren hat sich im Alltag als noch komplexer erwiesen als es für die soliden Tumoren gilt.

Therapiekontrolle | Eine Behandlung von Krebserkrankungen mit Angiogenese-Inhibitoren führt häufig zu Nekrosen des Tumors, ohne dass eine deutliche Größenabnahme zu beobachten ist. Daher sind funktionelle Bildgebungsverfahren hier besonders geeignet, um frühzeitig das Ansprechen auf die Therapie zu beurteilen. Für diese Aufgabe sind sowohl RECIST- als auch die Kriterien der WHO, die sich auf die Tumorgröße beziehen, nicht geeignet $[8,9]$.

\section{Nebenwirkungen und Sicherheit}

Akute Nebenwirkungen | Bei den Nebenwirkungen sind akute, späte und sehr späte Nebenwirkungen zu unterscheiden. Akute Nebenwirkungen treten innerhalb einer Stunde nach Kontrastmittelgabe auf. Hierbei handelt es sich um Überempfindlichkeitsreaktionen, die sowohl nach Iod- und Gadolinium-Kontrastmitteln wie auch nach Ultraschallkontrastmitteln beobachtet wurden. Die Inzidenz ist nach Iodkontrastmitteln am höchsten und nach Ultraschallkontrastmitteln am geringsten [13]. Im klinischen Alltag sind akute Überempfindlichkeitsreaktionen bei 1 von 10000 Untersuchungen beobachtet worden.

Späte Nebenwirkungen | Diese Reaktionen treten zwischen einer Stunde und einer Woche nach Injektion auf. Dies sind vor allem Hautreaktionen, die nach Iod-Kontrastmittel aber nicht nach Gadolinium- und Ultraschallkontrastmittel beschrieben wurden [13]. Sehr späte Nebenwirkungen treten nach über einer Woche nach Kontrastmittelgabe auf. Dabei handelt es sich um

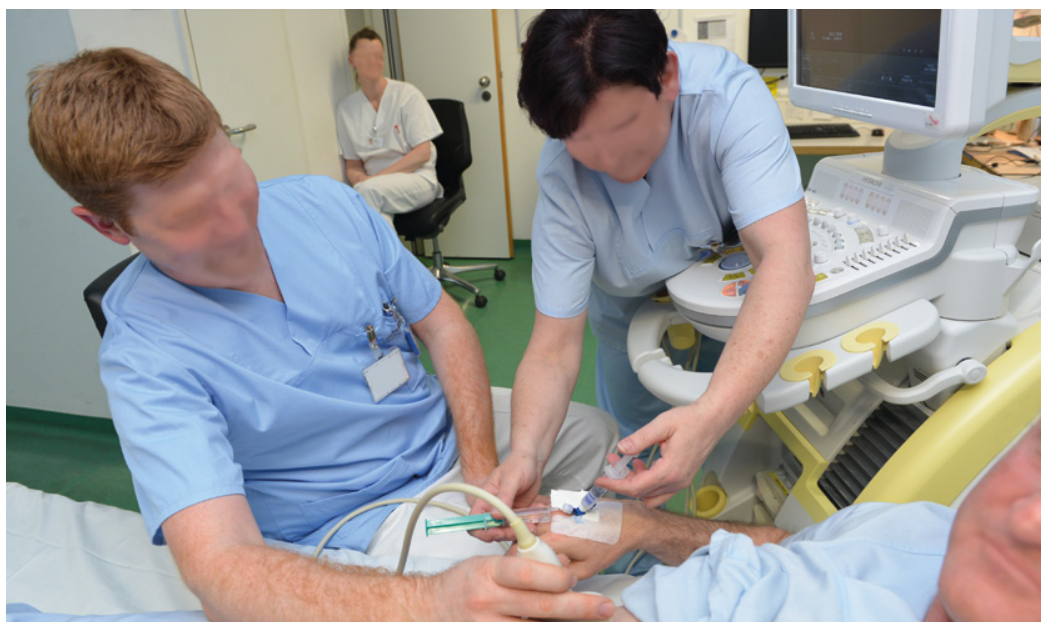

Abb. 7 In dieser Abbildung sind typische Fehler gezeigt. Die dünne Verweilkanüle ist auf dem Handrücken positioniert, was die Anflutung des KM erschwert. Die Kanüle liegt ausserdem auf der rechten Seite, so dass die Assistenz bei der Injektion dem Untersucher im Weg steht.

Thyrotoxikose nach Gabe von Iod-Kontrastmitteln und Nephrogene Systemische Fibrose (NSF) nach Gabe von Gadolinium-Kontrastmitteln.

Darüber hinaus gibt es noch renale Nebenwirkungen nach lod- und GadoliniumKontrastmittelgabe, die als Kontrast-induzierte Nephropathie (CIN) bezeichnet werden.

Sicherheit | Insgesamt sind Ultraschallkontrastmittel sichere Substanzen mit einer sehr geringen Inzidenz von Nebenwirkungen. Kardio-, hepato- oder nephrotoxische Effekte sind nicht bekannt. Daher sind Laboruntersuchungen zur Beurteilung der Leber- und/oder Nierenfunktion vor der Anwendung nicht notwendig [8, 9]. Jede Entscheidung zur Verabreichung von Ultraschallkontrastmitteln und Durchführung entsprechender Maßnahmen sollte in Kenntnis der geltenden regulatorischen Bestimmungen getroffen werden. Kontraindikationen der Methode müssen dem Untersucher bekannt sein.

Anaphylaktoide Reaktion | Die seltene und nicht vorhersehbar anaphylaktoide (pseudoallergische) Reaktion auf Ultraschall-Kontrastmittel entspricht der anaphylaktoiden Reaktion auf andere kolloidale bzw. kristalline Suspensionen und lipid (liposomal)-umhüllte Therapeutika (C activation-related pseudoallergy, CARPA). Dieser anaphylaktoiden (pseudoallergischen) Reaktion liegt eine Komplement-Aktivierung zugrunde, welche vermutlich durch pulmonale perivaskuläre Makrophagen (PIMs) ausgelöst wird [14-17]. Dabei treten typischerweise Engegefühl in Brust und Hals sowie arterielle Hypotonie, Tachykardie und andere auf eine Allergie weisende Symptome auf. Der (präventive) Einsatz von Glukokortikoiden und Antihistaminika wird empfohlen. Die Therapie entspricht der des anaphylaktischen Schocks [3]. 


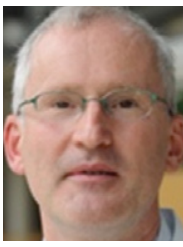

Prof. Dr. med. Christoph F. Dietrich

ist Chefarzt der Medizinischen Klinik 2 für Gastroenterologie und Stoffwechsel, Hämato-Onkologie, Geriatrie und Palliativmedizin am Caritas Krankenhaus Bad Mergentheim. christoph.dietrich@ckbm.de

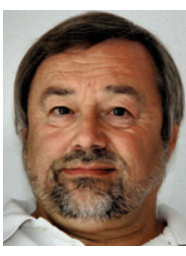

Dr. rer. nat. Christian Greis ist bei der Fa. Bracco als Leiter des globalen strategischen Marketings Ultraschall beschäftigt und ist Autor von zahlreichen Publikationen und Fachbuchartikel zum Thema Ultraschallkontrastmittel.

christian.greis@bracco.com

\section{Interessenkonflikt}

CG ist Mitarbeiter der Fa. Bracco, die das Ultraschallkontrastmittel SonoVue herstellt und vertreibt. CD gibt an, dass kein Interessenkonflikt besteht.

DOI 10.1055/s-0042-107959 Dtsch Med Wochenschr 2016; 141: 1019-1024 (c) Georg Thieme Verlag KG . Stuttgart · New York . ISSN 0012-0472
Allgemeine Empfehlungen

- Wie bei jeder Ultraschalldiagnostik sollte der Untersucher darauf achten, dass der gewählte mechanische Index so niedrig wie möglich gehalten wird und übermäßig lange Expositionszeiten vermieden werden.

- Die Verwendung von USKM bei Patienten mit schwerer koronarer Herzerkrankung sollte besonders sorgfältig geschehen.

- Wie auch bei der Verwendung anderer Kontrastmittel muss die Ausrüstung für eine Reanimation verfügbar sein.

- Die Verwendung von USKM sollte 24 Stunden vor einer extrakorporalen Stoßwellentherapie vermieden werden.

Mögliche Fehler | Fehlermöglichkeiten entstehen insbesondere

- bei falscher Geräteeinstellung oder Bedienung (zu hoher mechanischer Index mit Bläschenzerstörung und Schallabschwächung, eingeschränkte Signaltrennung von Gewebe und Kontrastmittelsignal),

- Unkenntnis von Artefakten sowie

- bei vorzeitiger Beendigung der Untersuchung. Fehldiagnosen bei der Kontrastmittelsonografie entstehen vorwiegend aufgrund unzureichender Kenntnisse und Erfahrungen des Untersuchers [3-5, 7]. Typische Fehler sind:

- falscher Arm (Untersucher- und Geräteseite)

- Venenverweilkanüle auf dem Handrücken ( Abb. 7) (weniger reproduzierbare Kreislaufzeiten im Vergleich zur Verweilkanüle in der Ellenbeuge)

- zu kleiner Diameter der Venenverweilkanüle (potenziell Bläschenzerstörung durch zu geringen Diameter)

- 3-Wegehahn (Änderung der Flussgeschwindigkeit durch unterschiedliche Lumendiameter)

Literatur

1 Albrecht T, Blomley M, Bolondi L et al. Guidelines for the use of contrast agents in ultrasound. January 2004. Ultraschall Med 2004; 25: 249-256

2 Dietrich CF, Schreiber-Dietrich D, Schuessler G, Ignee A. [Contrast enhanced ultrasound of the liver--state of the art]. Dtsch Med Wochenschr 2007; 132: 1225-1231

3 Frohlich E, Jenssen C, Schuler A, Dietrich CF. [Contrast-enhanced ultrasound for characterisation of focal liver lesions, practical advice]. Z Gastroenterol 2015; 53: 1099-1107

4 Dietrich CF, Ignee A, Hocke $M$ et al. Pitfalls and artefacts using contrast enhanced ultrasound. Z Gastroenterol 2011; 49: 350-356

5 Dietrich CF, Ignee A, Greis C et al. Artifacts and pitfalls in contrast-enhanced ultrasound of the liver. Ultraschall Med 2014; 35: 108-125

6 Dietrich CF, Frey H, Greis C. Grundlagen. In: Dietrich CF, editor. Ultrschall-Kurs. Köln: Deutscher Ärzte-Verlag, 2012: 7-50

7 Cui XW, Ignee A, Hocke $\mathrm{M}$ et al. Prolonged Heterogeneous Liver Enhancement on ContrastEnhanced Ultrasound. Ultraschall Med 2014; 35: 246-252
- Verlängerungsschnur, die alleine das Volumen des injizierten Kontrastmittels beinhaltet (mehr als 2,4 ml) ( Abb. 7).

- Unhandlichkeit durch Gerät, Assistenz und Untersucher (Assistenz hantiert zwischen Untersucher und Patient)

- Injektion von SonoVue durch den Filter der Venenverweilkanüle mit Zerstörung der Gasbläschen

Konsequenz für Klinik und Praxis

- In Deutschland ist nur SonoVue ${ }^{\circledR}$ für die Untersuchung der Leber zugelassen.

- Eine höhere oder zu hohe Kontrastmitteldosierung führt zu besseren Kontrasteffekten in der Spätphase und kompensiert Bläschenzerstörung teilweise.

- Allerdings führt dies zu Abschattung in der Tiefe, Überstrahlung kleiner Strukturen sowie das Erreichen einer Signalsättigung.

- Die Injektion kann wiederholt werden, wenn die meisten Mikrobläschen verschwunden sind und das KMUS-Bild wieder nahezu schwarz erscheint.

- In jeder Phase sollten aussagekräftige Videosequenzen gespeichert werden.

- Läsionen in der Leber werden in der Regel in der portalvenösen bzw. Spätphase detektiert. Bei anderen Organen wie z.B. der Prostata unterscheidet man keine arteriellen und venösen Phasen, da die Anflutung immer arteriell ist.

- Bei den Nebenwirkungen sind sehr seltene, akut auftretende allergoide Reaktionen zu beachten.
8 Claudon M, Dietrich CF, Choi Bl et al. Guidelines and good clinical practice recommendations for Contrast Enhanced Ultrasound (CEUS) in the liver - update 2012: A WFUMB-EFSUMB initiative in cooperation with representatives of AFSUMB, AIUM, ASUM, FLAUS and ICUS. Ultrasound Med Biol 2013; 39: 187-210

9 Claudon M, Dietrich CF, Choi Bl et al. Guidelines and good clinical practice recommendations for contrast enhanced ultrasound (CEUS) in the liver - update 2012: a WFUMB-EFSUMB initiative in cooperation with representatives of AFSUMB, AIUM, ASUM, FLAUS and ICUS. Ultraschall Med 2013; 34: 11-29

10 Jaspers N1, Pfister R, Kinkel H et al, Kontrastmittelsonographie, Dtsch Med Wochenschr. 2012;137: 2336-2339

Vollständiges Literaturverzeichnis unter http://dx.doi.org/10.1055/s-0042-107959 
11 Hocke M, Cui XW, Domagk D et al. Pancreatic cystic lesions: The value of contrast-enhanced endoscopic ultrasound to influence the clinical pathway. Endosc Ultrasound 2014; 3: 123-130

12 Dietrich C.F., Hocke M. Endosonographie zystischer Pankreasläsionen. Viszeralmedizin 2011; 27:

194-204

13 ESUR Contrast Media Safety Committee (CMSC) ESUR Guidelines on Contrast Media 9.0

14 Piscaglia F, Bolondi L. The safety of Sonovue in abdominal applications: retrospective analysis of 23188 investigations. Ultrasound Med Biol 2006; 32: $1369-1375$

15 Szebeni ], Muggia F, Gabizon A, Barenholz Y. Activation of complement by therapeutic liposomes and other lipid excipient-based therapeutic products: prediction and prevention. Adv Drug Deliv Rev 2011; 63: 1020-1030

16 Szebeni J, Baranyi L, Savay S et al. Role of complement activation in hypersensitivity reactions to doxil and hynic PEG liposomes: experimental and clinical studies. J Liposome Res 2002; 12: 165-172

17 Kitzman DW, Goldman ME, Gillam LD et al. Efficacy and safety of the novel ultrasound contrast agent perflutren (definity) in patients with suboptimal baseline left ventricular echocardiographic images. Am J Cardiol 2000; 86: 669-674 